\title{
Electric field and carrier concentration distributions in the semiconductor under photorefractive Gunn effect
}

\author{
P.M. Gorley, P.P. Horley, S.M. Chupyra \\ Yu. Fed'kovich Chernivtsi National University \\ 2, Kotsyubynsky str., 58012 Chernivtsi,Ukraine; e-mail: semicon@chnu.cv.ua
}

\begin{abstract}
In the framework of the one-dimension field model of semiconductor simultaneosly subjected to the action of carrier-warming electric field and two quasimonochromatic light waves the authors have numerically calculated the spatial-temporal distributions of inner electric field $E(x, \tau)$ and conductivity band electrons $n(x, \tau)$ in dependence on external control parameters (intensity of the incident light waves, their wave vector, external electric field and doping impurity concentration). It was found that the device operating on the base of photorefractive Gunn effect may be controllably switched between three following operation modes: low- and high-light wave intensity as well as a transition mode. The influence of the external control parameters on the $E(x, \tau)$ distribution was determined for each mode in question. It was shown that one could efficiently control the refractive index increment $\Delta n$ by means of proper change of the control parameters.
\end{abstract}

Keywords: compensated semiconductor, drift instability, photorefractive Gunn effect, refraction index.

Manuscript received 15.11.05; accepted for publication 15.12.05.

\section{Introduction}

The photorefractive effect [1] and Gunn effect [2] are both well-known and widely used in the different fields of electronics and sensorics. In 1996, the authors of the paper [3] predicted a new non-linear optical effect photorefractive analog of the Gunn effect (photo-Gunn effect, PhGE), representing a superposition of photorefractive and Gunn effects. In comparison with them, PhGE offered additional ways to control the domain propagation process through the semiconductor media by means of incident light wave intensity, and to control the photorefractive coefficient by the warming electric field. In the meantime, the investigation of the photo-Gunn effect passes its initial stage. In particular, the paper [4] shows that the presence of in-phase interference structures generated by two quasimonochromatic waves incident on the crystal leads to the formation of high-field domains. Papers [5] and [6] reported investigation results regarding the selforganization phenomena taking place under the PhGE in GaAs semiconductor. To solve the model describing PhGE, the authors of the original paper [3] used the expansion of the spatial-temporal electron and field distributions in the sample over trigonometric functions. They derived the analytical formulas for the phase variable increments regarding their stationary values, considering only the first harmonics of the expansion. At the same time, the influence of the control parameters (such as the intensity and frequency of the incident light wave, doping impurity concentration, carrier-warming electric field) on the time- and coordinate-dependent carrier concentrations and field distributions were not investigated as well as the convergence problem for the expansion used was considered. This paper is aimed at the clarification of the both points mentioned, which will contribute to the fundamental knowledge on the physics of non-equilibrium phenomena in semiconductors and, from the applied point of view, will favour the development of recommendations regarding the improvement of the technical characteristics of ultrahigh frequency electronic devices (Gunn diodes), sensors for the visible optical range, etc.

\section{Theoretical model}

In this study, the authors used the model proposed in the paper [3] to describe the carrier system under the existence of PhGE. Let us consider partiallycompensated semiconductor with the structure of $n$ GaAs, subjected simultaneously to carrier-warming electric field and two quasi-monochromatic light waves with slightly different frequencies $\omega_{0}$ and $\omega_{0}+\omega\left(\omega<<\omega_{0}\right)$, 
forming a travelling interference structure on the surface of the sample with the intensity $I(z, t)=$ $I_{0}[1+m \cos (K z+\omega t)]$, where $K=2 \pi / \Lambda$ is the wave number, $\Lambda$ is the distance between the peaks of $I(z, t), m$ is the modulation depth, and $I_{0}$ is the time-averaged wave intensity. Changes of the conductivity band electrons concentration $n$, ionized donors $N_{D}{ }^{i}$ and electric field $\mathbf{E}$ with time $t$ and space coordinate $z$ are described in the framework of the one-dimensional field model by the set of partial differential equations (including continuity and Poisson equations), written in dimensionless variables as [5]:

$$
\begin{aligned}
& \frac{\partial y_{1}}{\partial \tau}=a \cdot(1+m \cdot \cos (k x+\omega \tau)) \cdot\left(b-y_{1}\right)-y_{1} y_{2}, \\
& \frac{\partial y_{2}}{\partial \tau}=\frac{\partial y_{1}}{\partial \tau}+\alpha \frac{\partial}{\partial x}\left[y_{2} v+\beta \frac{\partial y_{2}}{\partial x}\right], \\
& \frac{\partial y_{3}}{\partial x}=-\frac{1}{a \beta}\left(y_{2}-y_{1}+1\right),
\end{aligned}
$$

with the parameters $y_{1}=N_{D}{ }^{i} / N_{A}, y_{2}=n / N_{A}, y_{3}=E / E_{s}$, $\tau=\gamma N_{A} t, \quad x=\varepsilon \varepsilon_{0} E_{s} \gamma z /(e D), \quad a=s I_{0} /\left(\gamma N_{A}\right)$, $b=N_{D} / N_{A}, \quad \alpha=\varepsilon \varepsilon_{0} E_{s} v_{s} /\left(e D N_{A}\right), \quad \beta=\varepsilon \varepsilon_{0} E_{s} \gamma /\left(e v_{s}\right)$, $k=e D K /\left(\varepsilon \varepsilon_{0} E_{s} \gamma\right)$, and dimensionless electron drift velocity $v \equiv v\left(y_{3}\right) / v_{s}=y_{3}\left(1+A y_{3}{ }^{3}\right) /\left(1+A y_{3}{ }^{4}\right)$. Here $s$ is the transversal ionization cross-section, $\gamma$ and $D$ - recombination and diffusion coefficients; $e$ is the elementary charge, $\varepsilon_{0}$ and $\varepsilon$ - dielectric constants for the vacuum and the semiconductor, respectively; $v_{s}$ is the saturation value of electron drift velocity, $E_{s}$ is the saturation field; and $A$ is the parameter depending on material properties. It is assumed that the coefficients $D, s$ and $\gamma$ do not depend on both electric field and coordinate.

As it was shown in [5], the stationary spacehomogeneous $(x \rightarrow \infty, \tau \rightarrow \infty)$ solutions of the system (1) $y_{10}$ and $y_{20}$ are

$$
\begin{aligned}
& y_{10}=0.5\left(\sqrt{(a-1)^{2}+4 a b}-(a-1)\right), \\
& y_{20}=0.5\left(\sqrt{(a-1)^{2}+4 a b}-(a+1)\right) .
\end{aligned}
$$

Stationary value of the electric field $y_{30}$ in the sample under the given electric current density $j_{0}$ can be found solving the equation $j_{0}=y_{20} v\left(y_{30}\right)$.

Let us look for the solution of the partial differential equations set (1) in the form of the expansion [7]

$$
y_{n}=y_{n 0}+\sum_{j=1}^{p}\left(\delta y_{n j}^{r e}(\tau) \cos (j k x)+\delta y_{n j}^{i m}(\tau) \sin (j k x)\right)
$$

$(n=1,2,3)$

with the weight coefficients $\delta y_{n j}^{r e, i m}$ depending on time but not the coordinate.

Considering three harmonics $(p=3)$ in the formula (3) and substituting it into (1), and further collecting the corresponding items by the sine and cosine of the same arguments, one will obtain the system of 12 equations regarding the coefficients $\delta y_{n j}^{r e, i m}$ of the general form

$$
\begin{aligned}
& \frac{\partial \delta y_{1 j}^{r e, i m}}{\partial \tau}=\operatorname{am}\left(b-y_{10}\right) \Psi\left(y_{1 j}^{r e, i m}, \cos (\omega \tau), \sin (\omega \tau)\right)+ \\
& +\prod_{j} h_{j}^{1} y_{1 j}^{r e, i m}+\prod_{i j} h_{i j}^{2} y_{1 i}^{r e} y_{2 j}^{i m}, \\
& \frac{\partial \delta y_{2 j}^{r e, i m}}{\partial \tau}=f(\alpha, \beta, k)\{\Theta(\alpha, \beta, k, a, m, b, \cos (\omega \tau), \sin (\omega \tau))+ \\
& +\prod_{j} h_{j}^{3} y_{p j}^{r e, i m}+\prod_{i j} h_{i j}^{4} y_{p i}^{r e} y_{p j}^{i m}+\prod_{i j j_{1}} h_{i j j_{1}}^{5} y_{p i}^{r e} y_{p j}^{i m} y_{p j_{1}}^{r e, i m}+ \\
& \left.+\prod_{i j i_{1} h_{1}} h_{i j j_{1} j_{1}}^{6} y_{p i}^{r e} y_{p j}^{i m} y_{p i_{1}}^{r e} y_{p j_{1}}^{i m}\right\},
\end{aligned}
$$

with the complex functions $\Psi$ and $\Theta$, and the coefficients $h_{j}^{1}, h_{i j}^{2}, h_{j}^{3}, h_{i j}^{4}, h_{i j j_{1}}^{5}, h_{i j i_{1} j_{1}}^{6}$, representing a certain combinations of $y_{10}, y_{20}, y_{30}, \alpha, \beta, k, a, m$, $\cos (\omega \tau)$, and $\sin (\omega \tau)$. The variables $\delta y_{j j}^{r e, i m}$ depend on $\delta y_{1 j, 2 j}^{r e, i m}$ according to the following expressions:

$\delta y_{3 j}^{r e}=\frac{1}{j} \frac{\delta y_{2 j}^{i m}-\delta y_{1 j}^{i m}}{\alpha \beta k}, \quad \delta y_{3 j}^{i m}=\frac{1}{j} \frac{\delta y_{1 j}^{r e}-\delta y_{2 j}^{r e}}{\alpha \beta k}$.

Let us emphasize that the formulas (5) satisfy the condition of electric neutrality of the crystal. In the addition to (4) and (5), one will obtain also three complementary equations for the non-trigonometric components regarding the variables $\delta y_{n j}^{r e, i m}$, which were used to verify the correctness of the numerical calculations.

\section{Calculation results and discussions}

The system of equations (4) was solved numerically with the $4^{\text {th }}$ order Runge-Kutta method [8] using the following material parameters for $n$-GaAs [1] (at $T=300 \mathrm{~K}): \varepsilon=13.2, \mu=0.5 \mathrm{~m}^{2} \mathrm{~V}^{-1} \mathrm{~s}^{-1}, v_{s}=8.5 \cdot 10^{4} \mathrm{~m} / \mathrm{s}$, $E_{s}=1.7 \cdot 10^{5} \mathrm{~V} / \mathrm{m}, \quad \gamma=10^{-12} \mathrm{~s}^{-1}, \quad D=0.0129 \mathrm{~m}^{2} / \mathrm{s}$, $N_{a}=10^{22} \mathrm{~m}^{-3}, A=0.04$.

It is necessary to emphasize that we have performed the calculations for three cases taking into account one, two and three harmonics of the expansion (3). Comparison between the obtained data sets has proven good convergence of the series expansion used. In particular, consideration of the next higher-order harmonic resulted only in the negligible (less than $2 \%$ ) changes to the dynamical variable values.

The obtained formulas (4) and (5) made it possible to calculate time and space distributions of the band electron concentration $n$ and electric field $E$ in the 
sample as a function of the following control parameters: intensity of the incident light wave $a$, frequency mismatch of the quasi-monochromatic waves $\omega$, their wavenumber $k$, modulation depth $m$, donor doping impurity concentration $b$ and external electric field $y_{30}$.

In Fig. 1, we have presented time-space surface plot of the electric field $y_{3}(x, \tau)$ for the fixed set of the control parameters $\left(k=34, m=0.1, \omega=10^{-4}, y_{30}=3, a=10^{-4}\right.$, $b=25)$. As expected, it has a well-pronounced planewave shape. As it turned out, the increase of the frequency mismatch value $\omega$ does not influence the oscillation amplitude, but leads to the increase of their frequency. At the same time, the amplitude grows linearly with the increase of $y_{30}$ (within the negative differential conductivity ranges $\left.2.5<y_{30}<3.5\right)$ and $m$ (for $0<m<0.5$ ). The spatial-temporal distribution of $y_{2}(x, \tau)$ also has a plane-wave character, but oscillations of the carrier concentration advance those of the electric field by $\pi / 2$.

Fig. 2 presents the surface plot $y_{3}(k, a)$ calculated for the sample with the normalized thickness $x=4.25$ for the fixed time $\tau=500$. As one can see, this surface features three different operation modes depending on the value of $a$ : low intensity mode $\left(a<3 \cdot 10^{-6}\right)$, high intensity mode $\left(a>1.3 \cdot 10^{-5}\right)$, and the transition one $\left(3 \cdot 10^{-6}<a<1.3 \cdot 10^{-5}\right)$. For these two former modes, $y_{3}(k)$ curves represent two travelling waves with almost the same amplitude, but phase-shifted by $\pi / 2$. In the transition region, the oscillation character changes smoothly from that of low-intensity to high-intensity mode. As it was found, both position and width of the transition region regarding the incident light intensity axis depend on the concentration of doping impurity and the external electric field, namely: for the increasing $b$ and constant $y_{30}$, the beginning of the transition region becomes shifted towards the lower intensities. Increase of $y_{30}$ within the ranges of the negative differential conductivity for the constant $b$ results in decreasing the transition region width.

It is worth noting that the electron concentration distribution $y_{2}(k, a)$ features the same three regions corresponding to different intensities plus a transition region, with the same phase shift of $\pi / 2$ between the $y_{2}(k, a)$ oscillations in low- and high-intensity modes and their smooth mutual transformation in the transition region. The difference between $y_{2}(k, a)$ and $y_{3}(k, a)$

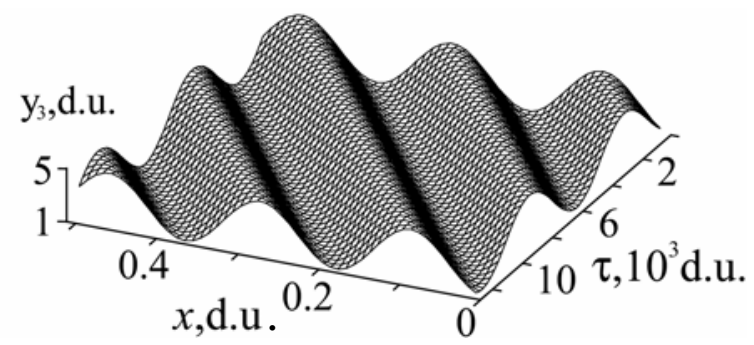

Fig. 1. Surface plot of the field phase variable $y_{3}(x, \tau)$.

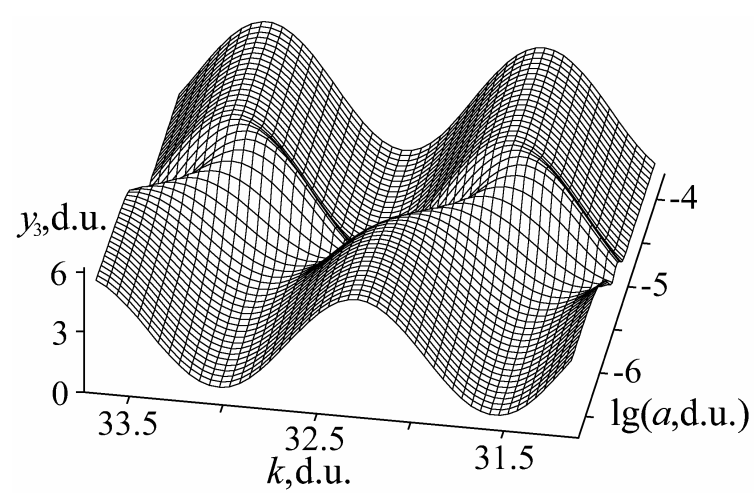

Fig. 2. Surface plot of $y_{3}(k, \lg a)$, calculated for $x=4.25$ and $\tau=500$.

surfaces can be seen in low- and high-illumination intensity modes, where the mean field value remains practically constant, while carrier concentration grows with the increase of the parameter $a$. At the same time, the growing speed of $y_{2}(a)$ curve in the high-intensity mode is significantly larger than that for the low-light intensities.

It is important that in both low- and high-intensity modes one can obtain almost equal maximum values of electric field $y_{3}$ by choosing the proper value of $k$. This result is of the significant application interest. As we know, $y_{3}(x, \tau, k, a)$ distribution determines the time- and space-periodical changes of the refraction index $\Delta n(x, \tau)=-0.5 n^{3} r_{41} E_{s} y_{3}(x, \tau)$ [1], where $n$ and $r_{41}$ are refractive and electrooptical coefficients, respectively. On the other hand, the same distribution influences the value of $\Delta n$ by means of the parameters $k$ and $a$. As it follows from the results of our numerical calculations, the minimal values of $\Delta n$ for $n$-GaAs presented in the paper [3] appear to be slightly underestimated, i.e. they are 1.3 to 1.5 and 3 to 4 times smaller for the high- and low-intensity modes, respectively. Such a difference can be attributed to the approximate character of the formulas (18) and (22) in the paper [3].

\section{Conclusions}

This paper presents the investigation results regarding the spatial-temporal distributions of the internal electric field and conductivity electron concentration as a function of the system control parameters (i.e., the incident light wave intensity, wave vector, external electric field and doping impurity concentration). It was shown that both low- and high-intensity modes are characterized with a travelling-wave form of the curve $y_{3}(k)$ with almost the same amplitude but phase-shifted by $\pi / 2$. The starting point of the transition region between these modes is determined by the concentration of donor doping impurity, while its width can be controlled using the applied electric field. As it follows 
from our investigations, the character of $y_{3}(x, \tau, k, a, b$, $y_{30}$ ) dependence, and hence the values of integral characteristics of the resulting device, operating on photorefractive Gunn effect (in particular, the refractive index increment $\Delta n$ ), can be efficiently controlled by the proper choice of the control parameter values $k, a, b$ and $y_{30}$. Our estimations have shown that the influence of the latter on the dynamics of the system can be significant and therefore experimentally detectable, which agrees well with the conclusions of the paper [3]

This investigation was performed in the framework of the research project GP/F11/0036 (Grant of the President of Ukraine for the support of research work of young scientists, 2006).

\section{References}

1. A. Yariv, P. Yen, Optical waves in crystals. Mir, Moscow (1987) (in Russian).
2. M.E. Levinshein, Yu.K. Pozhela, M.S. Shur, Gunn effect. Sovetskoye radio, Moscow (1975) (in Russian).

3. M. Segev, B. Collings, D. Abraham // Phys. Rev. Lett. 76, No 20, p. 3798-3801 (1996).

4. L.L. Bonilla, M. Kindelan, and P.J. Hernando // Phys. Rev. B 58, p. 7046-7052 (1998).

5. P.M. Gorley, P.P. Horley, J. Gonzalez-Hernandez, and Yu.V. Vorobiev // Materials Science and Engineering B 88, p. 286-291 (2002).

6. J. Gonzalez-Hernandez, Yu.V. Vorobiev, P.P. Horley, and P.M. Gorley // Modern Phys. Lett. B 15, No 17-19, p. 712-715 (2001).

7. P.P. Horley, Dynamical chaos and self-organization in the bipolar semiconductors with drift instability, Ph.D. dissertation. Chernivtsi State University, Chernivtsi, Ukraine, 1999.

8. E. Kamke, Handbook on ordinary differential equations. Mir, Moscow (1984) (in Russian). 